\title{
Níveis críticos foliares de nutrientes de três cultivares de batata-doce, selecionados para a produção de etanol ${ }^{1}$
}

\author{
Maurício Alberto Thumé $e^{2}$ Luiz Eduardo Dias ${ }^{3}$, Márcio Antônio da Silveira $^{4}$, Igor Rodrigues de Assis ${ }^{5}$
}

\section{RESUMO}

Nos últimos 12 anos, pesquisas desenvolvidas no Estado do Tocantins resultaram em dez novos cultivares de batata-doce com elevada concentração de amido, próprios para a produção de etanol. Entretanto, pouco se conhece a respeito de suas exigências nutricionais e teores foliares de nutrientes. Este trabalho teve como objetivo avaliar a absorção e a exportação de nutrientes e determinar os níveis críticos foliares de N, P e K, para os cultivares de batatadoce 'Amanda', 'Carolina Vitória' e 'Duda', em função de diferentes doses de N, P e K aplicadas ao solo. O experimento compreendeu 33 tratamentos, dispostos em delineamento de blocos casualizados, com quatro repetições. Os teores foliares de N, S, Cu e Zn variaram entre os cultivares e, os teores de $\mathrm{P}$ e K, apenas em função das doses de $\mathrm{P}_{2} \mathrm{O}_{5}$ e $\mathrm{K}_{2} \mathrm{O}$, respectivamente. Os teores de N, P e K nas raízes tuberosas variaram, significativamente, em função das doses destes nutrientes aplicadas ao solo, sendo que $\mathrm{K}$ foi o nutriente que se acumulou em maior quantidade, seguido do $\mathrm{N}$ e, este, de $\mathrm{Ca}, \mathrm{P}, \mathrm{Mg}$ e $\mathrm{S}$, cujas quantidades acumuladas foram cerca de dez vezes inferiores às do $\mathrm{N}$. De acordo com os níveis críticos foliares obtidos, o cultivar 'Amanda' mostrou-se mais exigente em $\mathrm{N} \mathrm{e} \mathrm{P} \mathrm{e,} \mathrm{o} \mathrm{cultivar} \mathrm{'Duda',} \mathrm{em} \mathrm{K,} \mathrm{indicando,}$ desta maneira, que as adubações com estes nutrientes devem ser individualizadas em função do cultivar, para que se atinja maior eficiência econômica e ambiental.

Palavras-chave: nutrição mineral, teor foliar, adubação, Ipomoea batatas (L.) Lam.

\section{ABSTRACT \\ Foliar nutrient critical levels in three cultivars of sweet potato selected for ethanol production}

In the past 12 years, research undertaken at Tocantins State, Brazil, resulted in ten new sweet potato cultivars with high starch content, suitable for ethanol production. However, little is known about their nutritional requirements and nutrient content. Thus, this study aimed to evaluate the uptake and exportation of nutrients and determine the leaf critical levels of N, P and K for the sweet potato cultivars 'Amanda', 'Carolina Victoria' and 'Duda', due to different doses of N, P and K applied to soil. The experiment comprised 33 treatments arranged in a completely randomized block design with four replications. Foliar concentrations of N, S, Cu and $\mathrm{Zn}$ varied among cultivars, while $\mathrm{P}$ and $\mathrm{K}$ varied due to $\mathrm{P}_{2} \mathrm{O}_{5}$ and $\mathrm{K}_{2} \mathrm{O}$ levels, respectively. The $\mathrm{N}, \mathrm{P}$ and $\mathrm{K}$ content in storage roots varied significantly depending on the doses of these nutrients in the soil, where $\mathrm{K}$ is the nutrient that has accumulated in greater quantity, followed by $\mathrm{N}$, and this one by $\mathrm{Ca}, \mathrm{P}, \mathrm{Mg}$ and $\mathrm{S}$, which accumulated amounts were about 10 times lower than the observed for N. According

\footnotetext{
Recebido para publicação em 22/06/2011 e aprovado em 04/07/2013.

${ }^{1}$ Trabalho extraído da Dissertação de Mestrado do primeiro autor.

${ }^{2}$ Engenheiro-Agrônomo, Mestre. Departamento de Agronomia, Universidade Federal do Tocantins, Campus Palmas, Avenida NS 15, ALCNO 14, Bloco IV, 109 Norte, Caixa Postal 266, 77001-090, Palmas, Tocantins, Brasil. mauricioagri@ hotmail.com

${ }^{3}$ Engenheiro-Agrônomo, Doutor. Departamento de Solos, Universidade Federal de Viçosa, Campus Viçosa, Avenida Peter Henry Rolfs, s/n, 36570-000, Viçosa, Minas Gerais, Brasil. Bolsista CNPq. ledias@ufv.br (autor para correspondência).

${ }^{4}$ Engenheiro-Agrônomo, Doutor. Departamento de Agronomia, Universidade Federal do Tocantins, Campus Palmas, Avenida NS 15, ALCNO 14, Bloco IV, 109 Norte, Caixa Postal 266,77001-090, Palmas, Tocantins, Brasil. marcio@uft.edu.br

${ }_{5}^{5}$ Engenheiro Agrícola e Ambiental, Doutor. Departamento de Solos, Universidade Federal de Viçosa, Campus Viçosa, Avenida Peter Henry Rolfs, s/n, 36570-000, Viçosa, Minas Gerais, Brasil. igor.assis@ufv.br
} 
to the leaf critical levels obtained, the cultivar 'Amanda' demands more $\mathrm{N}$ and $\mathrm{P}$, while 'Duda' needs more $\mathrm{K}$. These results indicate that the fertilization using these nutrients should be individualized, depending on the cultivar, in order to achieve greater economic and environmental efficiency.

Key words: mineral nutrition, leaf content, fertilization, Ipomoea batatas (L.) Lam.

\section{INTRODUÇÃO}

A batata-doce está entre as cinco principais culturas de 50 países e somente a China é responsável por $82,5 \%$ de toda a produção mundial. No Brasil, é cultivada praticamente em todo o território nacional, porém com a metade da produtividade média obtida pelos agricultores chineses (Silveira et al., 2007).

Por ser tradicionalmente cultivada por pequenos produtores e a maior parte dessa produção ser destinada ao mercado local, ou para consumo próprio, na agricultura familiar, a batata-doce não tem recebido grande atenção por parte da pesquisa agrícola brasileira, notadamente no que se refere ao conhecimento de suas demandas nutricionais e critérios de recomendação de adubação. No entanto, alguns trabalhos foram desenvolvidos, visando a avaliar a resposta de diferentes cultivares à adubação orgânica (Oliveira et al.; 2007; Oliveira et al., 2010 ; Santos et al., 2010) e aos fertilizantes com nitrogênio (Oliveira et al., 2005 e 2006; Alves et al., 2009; Foloni et al., 2013), fósforo (Oliveira et al., 2005 e 2006) e potássio e boro (Echer et al., 2009).

O programa de melhoramento vegetal para a batatadoce, desenvolvido pela Universidade Federal do Tocantins, lançou dez novos cultivares, voltados para a produção de etanol (Silveira et al., 2007). Apesar de um ganho efetivo em relação aos teores de amido nas raízes e da produtividade conseguida com os novos cultivares, até o momento, pouco ou quase nada é conhecido a respeito das exigências nutricionais e da distribuição de nutrientes entre a parte aérea e raízes, bem como dos níveis críticos foliares de N, P e K. Estima-se que a demanda por nutrientes, para produtividade da ordem de 11 a $15 \mathrm{t} \mathrm{ha}^{-1}$ de raízes tuberosas, exigiria cerca de 60 a $113 \mathrm{~kg} \mathrm{ha}^{-1} \mathrm{de} \mathrm{N}$, 20 a $47,5 \mathrm{~kg} \mathrm{ha}^{-1} \mathrm{de}_{2} \mathrm{O}_{5}, 100$ a $236 \mathrm{~kg} \mathrm{ha}^{-1}$ de $\mathrm{K}_{2} \mathrm{O}, 31$ a 35 $\mathrm{kg} \mathrm{ha}^{-1} \mathrm{de} \mathrm{CaO}$ e 11 a $13 \mathrm{~kg} \mathrm{ha}^{-1} \mathrm{de} \mathrm{MgO}$ (Silva et al., 2002), ou seja, quantidades elevadas de fertilizantes, principalmente de K e N, como também observado por Echer et al. (2009). A elevada demanda por K também foi relatada como a principal causa de declínio da produtividade da cultura, em solos intensamente cultivados, em Papua Nova Guiné (Bailey et al., 2009). A absorção de nutrientes em hortaliças segue um padrão de crescimento ou acúmulo de massa seca, sendo o K normalmente o mais absorvido (Ferreira et al., 1990), seguido do N, P, Ca e Mg (Embrapa, 1997).
Para o cultivar Canadense, o período de maior absorção de $\mathrm{N}$ e K pelas raízes tuberosas foi entre 115-145 e 115-130 dias após o transplantio, respectivamente (Echer et al., 2009).

A obtenção de cultivares de batata-doce de elevada produtividade (65,5 t ha ${ }^{-1}$, Silveira et al., 2007) e com raízes tuberosas de elevada concentração de amido (30,2\%, Silveira et al., 2007) é um avanço significativo para o desenvolvimento de alternativas para o programa brasileiro de biocombustíveis. No entanto, o conhecimento das demandas por nutrientes pelos cultivares permitiria o manejo mais racional de adubações, reduzindo custos, notadamente para nutrientes como o K, que apresenta elevada demanda por parte da cultura (Melvin et al., 2002), além de propiciar menor impacto ambiental.

Este trabalho foi conduzido com os objetivos de avaliar a absorção e a exportação de nutrientes e de determinar os níveis críticos foliares de N, P e K, para os cultivares de batata-doce 'Amanda', 'Carolina Vitória'e 'Duda'.

\section{MATERIAL E MÉTODOS}

O experimento foi conduzido na Estação Experimental da Universidade Federal do Tocantins, em Palmas-TO, em um Latossolo Vermelho-Amarelo distrófico (Tabela 1). O preparo da área experimental, que se encontrava em pousio (seis meses), iniciou-se com aração e gradagem, 30 dias antes do plantio. As leiras foram construídas mecanicamente, espaçadas de $0,90 \mathrm{~m}$ e com aproximadamente 0,30 $\mathrm{m}$ de altura. Os nutrientes aplicados foram incorporados na parte superior da leira por ocasião do plantio, a aproximadamente $0,05 \mathrm{~m}$ de profundidade. A adubação com $\mathrm{P}$ foi realizada integralmente no plantio, com K e N parcelados, igualmente, em três aplicações (plantio, 30 e 60 dias após o plantio).

O experimento foi montado em delineamento em blocos casualizados, com quatro repetições, sendo testados 33 tratamentos oriundos de uma matriz baconiana (Turrent, 1979) e compostos com três cultivares de batata-doce ('Amanda', 'Carolina Vitória'e 'Duda'), submetidos a quatro doses de $\mathrm{N}\left(0 ; 60 ; 120\right.$ e $240 \mathrm{~kg} \mathrm{ha}^{-1}$, na forma de ureia); de $\mathrm{P}\left(0 ; 75,150\right.$ e $300 \mathrm{~kg} \mathrm{ha}^{-1}$ de $\mathrm{P}_{2} \mathrm{O}_{5}$, na forma de superfosfato triplo) e de $\mathrm{K}\left(0 ; 75,150\right.$ e $300 \mathrm{~kg} \mathrm{ha}^{-1} \mathrm{de} \mathrm{K}_{2} \mathrm{O}$, na forma de cloreto de potássio), totalizando 132 parcelas experimentais (Tabela 2). 
A parcela experimental de $18,9 \mathrm{~m}^{2}(4,2 \mathrm{~m} \mathrm{x} 4,5 \mathrm{~m})$ foi composta por cinco linhas de plantio, contendo cada linha 14 plantas (espaçadas por $0,3 \mathrm{~m}$ ), totalizando 70 plantas. Considerou-se parcela útil a área das 30 plantas centrais de cada parcela.

Os cultivares avaliados foram obtidos pelo Programa de Melhoramento Genético desenvolvido pela UFT (Silveira et al., 2007), cujas características são:
- 'Amanda': cultivar com película externa branca, polpa creme, ciclo precoce (4-5 meses) e raízes com massas superiores a $500 \mathrm{~g}$. A produtividade média é de $46,7 \mathrm{t} \mathrm{ha}^{-1}$, e, o teor médio de amido, de 21,4\%, proporcionando rendimento médio de $141 \mathrm{Lt}^{-1}$ e produtividade de $6.595 \mathrm{~L} \mathrm{ha}^{-1} \mathrm{de}$ etanol;

- 'Carolina Vitória': cultivar de ciclo tardio (6-7 meses), película externa roxa e polpa branca. A produtividade

Tabela 1. Atributos químicos e análise granulométrica do Latossolo Vermelho-Amarelo distrófico $(0-20 \mathrm{~cm})$ da área experimental no campus da UFT, em Palmas - TO

\begin{tabular}{|c|c|c|c|c|c|c|c|c|c|c|c|c|}
\hline \multirow{3}{*}{$\begin{array}{l}\mathrm{pH} \\
\mathrm{CaCl}_{2}\end{array}$} & \multirow{2}{*}{$\mathbf{P}$} & \multirow{2}{*}{$\mathbf{K}$} & \multirow{2}{*}{$\mathrm{Ca}^{2+}$} & \multirow{2}{*}{$\mathbf{M g}^{2+}$} & \multirow{2}{*}{$\mathbf{A l}^{3+}$} & \multirow{2}{*}{$\mathbf{H}+\mathbf{A l}$} & \multirow{3}{*}{$\mathbf{T}$} & \multirow{2}{*}{$\mathbf{V}$} & \multirow{2}{*}{ MO } & \multicolumn{3}{|c|}{ Granulometria $^{1}$} \\
\hline & & & & & & & & & & Areia & Silte & Arg. \\
\hline & \multicolumn{2}{|c|}{$\mathrm{mg} \mathrm{dm} \mathrm{m}^{-3}$} & \multicolumn{4}{|c|}{$\mathrm{cmol}_{\mathrm{c}} \mathrm{dm}^{-3}$} & & $\%$ & $\overline{\mathrm{dag} \mathrm{kg}^{-1}}$ & & $\mathrm{~g} \mathrm{~kg}^{-1}$ & \\
\hline 4,2 & 0,8 & 104 & 1,8 & 1,0 & 0,1 & 6,1 & 9,2 & 33,5 & 1,4 & 680 & 80 & 240 \\
\hline
\end{tabular}

$\mathrm{P}$ e $\mathrm{K}$ disponíveis extraídos com Mehlich-1; Ca, $\mathrm{Mg}$ e Al trocáveis extraídos com $\mathrm{KCl} 1 \mathrm{~mol} \mathrm{~L}{ }^{-1}$; Acidez potencial a pH 7,0 extraída com acetato de cálcio $1 \mathrm{~mol} \mathrm{~L}^{-1}$. MO: matéria orgânica. ${ }^{1}$ Análise granulométrica: método da pipeta (Embrapa, 1997).

Tabela 2. Doses de $\mathrm{N}, \mathrm{P}_{2} \mathrm{O}_{5}$ e $\mathrm{K}_{2} \mathrm{O}$ testadas para cada cultivar de batata-doce e identificação dos respectivos tratamentos

\begin{tabular}{|c|c|c|c|c|c|}
\hline \multirow{3}{*}{ Tratamento } & \multirow{3}{*}{ Identificação } & \multirow{3}{*}{ Cultivar } & \multicolumn{3}{|c|}{ Dose } \\
\hline & & & $\mathbf{N}$ & $\mathbf{P}_{2} \mathbf{O}_{5}$ & $\mathrm{~K}_{2} \mathrm{O}$ \\
\hline & & & \multicolumn{3}{|c|}{$\mathrm{kg} \mathrm{ha}^{-1}$} \\
\hline 1 & $\mathrm{~N}_{0} \mathrm{P}_{0} \mathrm{~K}_{0}-\mathrm{A}$ & 'Amanda' & 0 & 0 & 0 \\
\hline 2 & $\mathrm{~N}_{0} \mathrm{P}_{150} \mathrm{~K}_{150}$ & 'Amanda' & 0 & 150 & 150 \\
\hline 3 & $\mathrm{~N}_{60} \mathrm{P}_{150} \mathrm{~K}_{150}$ & 'Amanda' & 60 & 150 & 150 \\
\hline $4^{1 /}$ & $\mathrm{N}_{120} \mathrm{P}_{150} \mathrm{~K}_{150}$ & 'Amanda' & 120 & 150 & 150 \\
\hline 5 & $\mathrm{~N}_{240} \mathrm{P}_{150} \mathrm{~K}_{150}$ & 'Amanda' & 240 & 150 & 150 \\
\hline 6 & $\mathrm{~N}_{120} \mathrm{P}_{0} \mathrm{~K}_{150}$ & 'Amanda' & 120 & 0 & 150 \\
\hline 7 & $\mathrm{~N}_{120} \mathrm{P}_{75} \mathrm{~K}_{150}$ & 'Amanda' & 120 & 75 & 150 \\
\hline 8 & $\mathrm{~N}_{120}^{120} \mathrm{P}_{300} \mathrm{~K}_{150}$ & 'Amanda' & 120 & 300 & 150 \\
\hline 9 & $\mathrm{~N}_{120} \mathrm{P}_{150} \mathrm{~K}_{0}$ & 'Amanda' & 120 & 150 & 0 \\
\hline 10 & $\mathrm{~N}_{120} \mathrm{P}_{150} \mathrm{~K}_{75}$ & 'Amanda' & 120 & 150 & 75 \\
\hline 11 & $\mathrm{~N}_{120} \mathrm{P}_{150} \mathrm{~K}_{300}$ & 'Amanda' & 120 & 150 & 300 \\
\hline 12 & $\mathrm{~N}_{0} \mathrm{P}_{0} \mathrm{~K}_{0}-\mathrm{C}$ & 'Carolina Vitória' & 0 & 0 & 0 \\
\hline 13 & $\mathrm{~N}_{0} \mathrm{P}_{150}^{0} \mathrm{~K}_{150}$ & 'Carolina Vitória' & 0 & 150 & 150 \\
\hline 14 & $\mathrm{~N}_{60} \mathrm{P}_{150} \mathrm{~K}_{150}$ & 'Carolina Vitória' & 60 & 150 & 150 \\
\hline 15 & $\mathrm{~N}_{120} \mathrm{P}_{150} \mathrm{~K}_{150}$ & 'Carolina Vitória' & 120 & 150 & 150 \\
\hline 16 & $\mathrm{~N}_{240}^{120} \mathrm{P}_{150} \mathrm{~K}_{150}^{150}$ & 'Carolina Vitória' & 240 & 150 & 150 \\
\hline 17 & $\mathrm{~N}_{120} \mathrm{P}_{0} \mathrm{~K}_{150}$ & 'Carolina Vitória' & 120 & 0 & 150 \\
\hline 18 & $\mathrm{~N}_{120} \mathrm{P}_{75} \mathrm{~K}_{150}$ & 'Carolina Vitória' & 120 & 75 & 150 \\
\hline 19 & $\mathrm{~N}_{120} \mathrm{P}_{300} \mathrm{~K}_{150}$ & 'Carolina Vitória' & 120 & 300 & 150 \\
\hline 20 & $\mathrm{~N}_{120} \mathrm{P}_{150} \mathrm{~K}_{0}$ & 'Carolina Vitória' & 120 & 150 & 0 \\
\hline 21 & $\mathrm{~N}_{120} \mathrm{P}_{150} \mathrm{~K}_{75}$ & 'Carolina Vitória' & 120 & 150 & 75 \\
\hline 22 & $\mathrm{~N}_{120} \mathrm{P}_{150} \mathrm{~K}_{300}$ & 'Carolina Vitória' & 120 & 150 & 300 \\
\hline 23 & $\mathrm{~N}_{0} \mathrm{P}_{0} \mathrm{~K}_{0}-\mathrm{D}$ & 'Duda' & 0 & 0 & 0 \\
\hline 24 & $\mathrm{~N}_{0} \mathrm{P}_{150} \mathrm{~K}_{150}$ & 'Duda' & 0 & 150 & 150 \\
\hline 25 & $\mathrm{~N}_{60} \mathrm{P}_{150} \mathrm{~K}_{150}$ & 'Duda' & 60 & 150 & 150 \\
\hline 26 & $\mathrm{~N}_{120} \mathrm{P}_{150} \mathrm{~K}_{150}$ & 'Duda' & 120 & 150 & 150 \\
\hline 27 & $\mathrm{~N}_{240} \mathrm{P}_{150} \mathrm{~K}_{150}$ & 'Duda' & 240 & 150 & 150 \\
\hline 28 & $\mathrm{~N}_{120}^{20} \mathrm{P}_{0} \mathrm{~K}_{150}$ & 'Duda' & 120 & 0 & 150 \\
\hline 29 & $\mathrm{~N}_{120} \mathrm{P}_{75} \mathrm{~K}_{150}$ & 'Duda' & 120 & 75 & 150 \\
\hline 30 & $\mathrm{~N}_{120} \mathrm{P}_{300} \mathrm{~K}_{150}$ & 'Duda' & 120 & 300 & 150 \\
\hline 31 & $\mathrm{~N}_{120} \mathrm{P}_{150} \mathrm{~K}_{0}$ & 'Duda' & 120 & 150 & 0 \\
\hline 32 & $\mathrm{~N}_{120} \mathrm{P}_{150} \mathrm{~K}_{75}$ & 'Duda' & 120 & 150 & 75 \\
\hline 33 & $\mathrm{~N}_{120} \mathrm{P}_{150} \mathrm{~K}_{300}$ & 'Duda' & 120 & 150 & 300 \\
\hline
\end{tabular}

1/ Tratamento referência. 
média é de $32,2 \mathrm{t} \mathrm{ha}{ }^{-1} \mathrm{e}$, o teor médio de amido, de 30,2\%, proporcionando rendimento médio de $199 \mathrm{~L} \mathrm{t}^{-1} \mathrm{e}$ produtividade de $6.412 \mathrm{~L} \mathrm{ha}^{-1}$ de etanol;

- 'Duda': cultivar de ciclo tardio (6-7 meses), película externa roxa e polpa branca. A produtividade média é de $65,5 \mathrm{t} \mathrm{ha}^{-1} \mathrm{e}$, o teor médio de amido, de $24,4 \%$, proporcionando rendimento médio de $161 \mathrm{~L} \mathrm{t}^{-1}$ e produtividade de 10.467 $\mathrm{L} \mathrm{ha}^{-1}$ de etanol.

O plantio foi realizado no mês de fevereiro, utilizandose segmentos de ramas de aproximadamente $0,25 \mathrm{~m}$, retirados no dia anterior, na área de multiplicação da própria estação experimental da UFT. Foi utilizada uma rama por cova, enterrada manualmente a uma profundidade aproximada de $0,10 \mathrm{~m}$. Aos 25 dias após o plantio, foi realizada a capina mecânica e o remonte das leiras. As invasoras restantes na parte superior da leira foram retiradas manualmente. Aos 60 dias, foi realizada a última eliminação das ervas, novamente por catação manual. Durante os primeiros 30 dias de cultivo, as parcelas foram irrigadas, conforme a demanda hídrica, em função das precipitações no período de condução do experimento, que foram da ordem de $722 \mathrm{~mm}$.

Aos 90 dias após o plantio, foram retiradas duas folhas totalmente expandidas e localizadas no terço médio das ramas de cada planta da área amostral (Jones et al., 1991). As folhas foram lavadas com água deionizada, secas em estufa de circulação de ar forçado, até peso constante, moídas, homogeneizadas e digeridas em solução nítrico-perclórica (3:1) e, posteriormente, determinados os teores totais de $\mathrm{P}, \mathrm{K}, \mathrm{Ca}, \mathrm{Mg}, \mathrm{S}, \mathrm{Zn}, \mathrm{Mn}, \mathrm{Cu}$ e Fe, por espectrometria de emissão óptica, com plasma indutivelmente acoplado - ICP-OES. A determinação do teor total de $\mathrm{N}$ das folhas foi realizada pelo método Kjeldahl (Bremner \& Mulvaney, 1982). Nesta mesma oportunidade, foram retiradas nove amostras simples $(0-20 \mathrm{~cm})$, da parte superior das leiras de cada parcela, para formar uma amostra composta, após homogeneização e quarteamento. As amostras foram encaminhadas para o Laboratório de Rotina de Fertilidade do Solo da Universidade Federal de Viçosa, para a determinação dos teores disponíveis de $\mathrm{P}$, $\mathrm{K}$ e S e trocáveis de $\mathrm{Ca}, \mathrm{Mg}$ e $\mathrm{Al}$.

Aos 180 dias de cultivo, as plantas de cada parcela amostral foram coletadas, separando-se as ramas, as folhas e as raízes tuberosas para a determinação da massa da matéria fresca. Amostras dos materiais vegetais de seis plantas foram retiradas, para a determinação da matéria seca, a $70{ }^{\circ} \mathrm{C}$, em estufa de circulação forçada de ar, até massa constante.

Os dados obtidos foram analisados estatisticamente, por meio de análise de variância e, por se tratar de uma matriz baconiana, não é possível o estudo de interação entre as variáveis independentes. $\mathrm{O}$ desdobramento dos efeitos foi realizado por meio de análise de regressão, ten- do os teores de $\mathrm{N}, \mathrm{P}$ e $\mathrm{K}$ nas folhas e nas raízes tuberosas como dependentes das doses de $\mathrm{N}, \mathrm{P}_{2} \mathrm{O}_{5}$ e $\mathrm{K}_{2} \mathrm{O}$ aplicadas ao solo. Da mesma forma, os teores de $\mathrm{P}$ e $\mathrm{K}$ disponíveis no solo foram analisados como variáveis dependentes das doses de $\mathrm{P}_{2} \mathrm{O}_{5}$ e $\mathrm{K}_{2} \mathrm{O}$ aplicadas ao solo. Os coeficientes das equações obtidas foram testados pelo teste $\mathrm{F}$, a $5 \%$ de probabilidade.

\section{RESULTADOS E DISCUSSÃO \\ Teores foliares de nutrientes aos 90 dias de cultivo}

Os teores de nutrientes das folhas, coletadas aos 90 dias após o plantio, variaram em função dos níveis de adubação e dos cultivares. Os menores teores de N, P e K para o cultivar 'Amanda' foram observados com as plantas das parcelas referente ao tratamento testemunha, que não recebeu adubação $\left(26,55 ; 1,23 ; 11,81 \mathrm{~g} \mathrm{~kg}^{-1}\right.$, respectivamente) (Tabela 3). Para o cultivar 'Carolina Vitória', o menor teor de $\mathrm{N}$ foi observado no tratamento $\mathrm{N}_{60} \mathrm{P}_{150} \mathrm{~K}_{150}$ $\left(28,13 \mathrm{~g} \mathrm{~kg}^{-1}\right)$; o de $\mathrm{P}$, no tratamento $\mathrm{N}_{120} \mathrm{P}_{150} \mathrm{~K}_{0}\left(1,28 \mathrm{~g} \mathrm{~kg}^{-1}\right)$ e, o de K, no tratamento $\mathrm{N}_{120} \mathrm{P}_{150} \mathrm{~K}_{300}\left(12,26 \mathrm{~g} \mathrm{~kg}^{-1}\right)$. Os menores teores foliares de $\mathrm{N}$ e $\mathrm{P}$ para o cultivar 'Duda' foram observados nas plantas do tratamento testemunha (24,85 e 1,15 $\mathrm{g} \mathrm{kg}^{-1}$, respectivamente), e, o de $\mathrm{K}$, no tratamento $\mathrm{N}_{60} \mathrm{P}_{150} \mathrm{~K}_{150}\left(10,06 \mathrm{~g} \mathrm{~kg}^{-1}\right)$. Ao se observarem os valores médios entre os tratamentos, as variações entre cultivares podem ser consideradas como de pequena magnitude, com exceção apenas para o teor foliar médio de $\mathrm{N}$ para o cultivar 'Duda'que foi cerca de $10 \%$ inferior ao observado para os outros cultivares (Tabela 3 ). Esse comportamento difere do observado por Melvin et al. (2002), que encontraram diferenças significativas na absorção e na eficiência de utilização de $\mathrm{K}$, por parte de diferentes genótipos de batata doce, na China. Ao estudarem o efeito de doses de $\mathrm{N}$ e de $\mathrm{K}$ no cultivar Canadense, Foloni et al. (2013) encontraram teores foliares que variam de 14 a $37 \mathrm{~g} \mathrm{~kg}^{-1}$ e de 8,5 a 13,6 $\mathrm{g} \mathrm{kg}^{-1}$, para $\mathrm{Ne} \mathrm{K}$, respectivamente. De acordo com Lorenzi et al. (1997), o intervalo de suficiência para $\mathrm{N}$ para a cultura da batata-doce seria de 33 a 45 $\mathrm{g} \mathrm{kg}^{-1}$; o de P, entre 2,3 e $5,0 \mathrm{~g} \mathrm{~kg}^{-1} \mathrm{e}$, o de $\mathrm{K}$, entre 31 e 45 $\mathrm{g} \mathrm{kg}^{-1}$. Os valores médios entre tratamentos, observados paras os três cultivares, ou estão muito inferiores, ou na base da faixa de suficiência proposta por aqueles autores (Tabela 3). Por outro lado, os teores foliares médios de N, Pe K, para 56 cultivos realizados na Índia (32,1; 2,3 e 23,2 $\mathrm{g} \mathrm{kg}^{-1}$, respectivamente, (Ramakrishna et al., 2009), estão mais próximos às concentrações obtidas neste trabalho.

Os teores foliares de $\mathrm{Ca}, \mathrm{Mg}$ e $\mathrm{S}$ também apresentaram variações, em função dos níveis de adubação e dos cultivares; no entanto, essas variações foram, de maneira geral, de menores magnitudes que as observadas para os demais macronutrientes (Tabela 3), observando-se dife- 
rença estatística significativa $(\mathrm{P}<0,05)$ apenas para $\mathrm{S}$, entre os cultivares, com menor teor médio para as folhas do cultivar 'Duda'. Da mesma forma como observado para N, $\mathrm{P}$ e $\mathrm{K}$, os teores foliares de $\mathrm{Ca}, \mathrm{Mg}$ e, principalmente, $\mathrm{S}$, situaram-se abaixo da faixa de suficiência proposta por Lorenzi et al. (1979). Esse resultado pode sugerir a ocorrência de deficiência nutricional; no entanto, deve-se considerar que a análise isolada da concentração do nutriente nas folhas pode não representar, necessariamente, a existência de deficiência, uma vez que, a avaliação das relações da concentração de cada nutriente em relação aos demais como no caso do sistema DRIS - tende a indicar melhor o estado nutricional das plantas por meio do equilíbrio entre nutrientes (Beaufils, 1973; Sumner, 1977).

Entre os micronutrientes analisados, observou-se diferença significativa entre cultivares apenas para $\mathrm{Cu}(\mathrm{p}<$ 0,01) e Zn ( $\mathrm{p}<0,05)$, sendo 'Carolina Vitória' o cultivar que apresentou valores médios significativamente supe-

Tabela 3. Teores médios de nitrogênio $(\mathrm{N})$, fósforo $(\mathrm{P})$, potássio $(\mathrm{K})$, cálcio $(\mathrm{Ca})$, magnésio $(\mathrm{Mg})$ e enxofre $(\mathrm{S})$ nas amostras de folhas dos cultivares 'Amanda', 'Carolina Vitória'e 'Duda', coletadas 90 dias após o plantio

\begin{tabular}{|c|c|c|c|c|c|c|}
\hline \multirow{3}{*}{ Tratamento } & $\mathbf{N}$ & $\mathbf{P}$ & $\mathbf{K}$ & $\mathbf{C a}$ & Mg & $\mathbf{S}$ \\
\hline & \multicolumn{6}{|c|}{$\mathrm{g} \mathrm{kg}^{-1}$} \\
\hline & \multicolumn{6}{|c|}{ 'Amanda' } \\
\hline$\overline{\mathrm{N}_{0} \mathrm{P}_{0} \mathrm{~K}_{0}}$ & 26,55 & 1,23 & 11,81 & 6,44 & 2,24 & 1,59 \\
\hline $\mathrm{N}_{0} \mathrm{P}_{150} \mathrm{~K}_{150}$ & 27,20 & 1,45 & 18,70 & 6,03 & 2,19 & 1,66 \\
\hline $\mathrm{N}_{60} \mathrm{P}_{150} \mathrm{~K}_{150}$ & 31,61 & 1,80 & 24,82 & 6,03 & 1,88 & 1,70 \\
\hline $\mathrm{N}_{120} \mathrm{P}_{150} \mathrm{~K}_{150}$ & 32,77 & 1,45 & 19,59 & 7,68 & 2,53 & 1,63 \\
\hline $\mathrm{N}_{240} \mathrm{P}_{150} \mathrm{~K}_{150}$ & 34,16 & 1,75 & 15,44 & 7,12 & 2,40 & 1,55 \\
\hline $\mathrm{N}_{120} \mathrm{P}_{0} \mathrm{~K}_{150}$ & 31,41 & 1,40 & 17,35 & 6,02 & 1,98 & 1,48 \\
\hline $\mathrm{N}_{120} \mathrm{P}_{75} \mathrm{~K}_{150}$ & 32,11 & 1,75 & 20,78 & 5,73 & 1,95 & 1,65 \\
\hline $\mathrm{N}_{120} \mathrm{P}_{300} \mathrm{~K}_{150}$ & 30,76 & 1,61 & 19,94 & 8,97 & 2,67 & 1,87 \\
\hline $\mathrm{N}_{120} \mathrm{P}_{150} \mathrm{~K}_{0}$ & 29,98 & 1,40 & 13,29 & 6,20 & 2,37 & 1,47 \\
\hline $\mathrm{N}_{120} \mathrm{P}_{150} \mathrm{~K}_{75}$ & 28,32 & 1,64 & 14,51 & 8,06 & 3,00 & 1,64 \\
\hline $\mathrm{N}_{120} \mathrm{P}_{150} \mathrm{~K}_{300}$ & 32,53 & 1,53 & 21,63 & 5,87 & 1,92 & 1,45 \\
\hline \multirow[t]{2}{*}{ Média } & 30,67 & 1,55 & 17,99 & 6,74 & 2,28 & 1,61 \\
\hline & \multicolumn{6}{|c|}{ 'Carolina Vitória' } \\
\hline $\mathrm{N}_{0} \mathrm{P}_{0} \mathrm{~K}_{0}$ & 28,83 & 1,34 & 17,45 & 8,28 & 3,57 & 1,70 \\
\hline $\mathrm{N}_{0} \mathrm{P}_{150} \mathrm{~K}_{150}$ & 29,17 & 1,61 & 18,86 & 8,37 & 3,87 & 1,95 \\
\hline $\mathrm{N}_{60} \mathrm{P}_{150} \mathrm{~K}_{150}$ & 28,13 & 1,68 & 23,73 & 6,99 & 2,64 & 1,82 \\
\hline $\mathrm{N}_{120} \mathrm{P}_{150} \mathrm{~K}_{150}$ & 32,19 & 1,96 & 20,83 & 7,47 & 3,00 & 1,94 \\
\hline $\mathrm{N}_{240} \mathrm{P}_{150} \mathrm{~K}_{150}$ & 33,62 & 1,40 & 13,34 & 7,51 & 2,97 & 1,37 \\
\hline $\mathrm{N}_{120} \mathrm{P}_{0} \mathrm{~K}_{150}$ & 31,88 & 1,49 & 28,72 & 5,35 & 2,29 & 1,78 \\
\hline $\mathrm{N}_{120} \mathrm{P}_{75} \mathrm{~K}_{150}$ & 29,98 & 1,66 & 24,34 & 8,17 & 3,21 & 1,84 \\
\hline $\mathrm{N}_{120} \mathrm{P}_{300} \mathrm{~K}_{150}$ & 30,02 & 1,65 & 26,27 & 8,49 & 3,42 & 1,75 \\
\hline $\mathrm{N}_{120} \mathrm{P}_{150} \mathrm{~K}_{0}$ & 30,91 & 1,28 & 18,15 & 10,50 & 3,97 & 1,47 \\
\hline $\mathrm{N}_{120} \mathrm{P}_{150} \mathrm{~K}_{75}$ & 31,41 & 1,75 & 18,06 & 7,88 & 3,26 & 1,84 \\
\hline $\mathrm{N}_{120} \mathrm{P}_{150} \mathrm{~K}_{300}$ & 29,75 & 1,64 & 12,26 & 6,53 & 2,77 & 1,76 \\
\hline \multirow[t]{2}{*}{ Média } & 30,54 & 1,59 & 19,27 & 7,78 & 3,18 & 1,75 \\
\hline & \multicolumn{6}{|c|}{ 'Duda' } \\
\hline $\mathrm{N}_{0} \mathrm{P}_{0} \mathrm{~K}_{0}$ & 24,85 & 1,15 & 15,93 & 7,79 & 3,19 & 1,48 \\
\hline $\mathrm{N}_{0} \mathrm{P}_{150} \mathrm{~K}_{150}$ & 26,39 & 1,41 & 22,80 & 8,29 & 3,10 & 1,61 \\
\hline $\mathrm{N}_{60} \mathrm{P}_{150} \mathrm{~K}_{150}$ & 27,59 & 1,35 & 10,06 & 6,36 & 2,67 & 1,27 \\
\hline $\mathrm{N}_{120} \mathrm{P}_{150} \mathrm{~K}_{150}$ & 29,52 & 1,46 & 26,01 & 6,32 & 2,44 & 1,37 \\
\hline $\mathrm{N}_{240} \mathrm{P}_{150} \mathrm{~K}_{150}$ & 30,72 & 1,45 & 19,68 & 7,41 & 3,12 & 1,18 \\
\hline $\mathrm{N}_{120} \mathrm{P}_{0} \mathrm{~K}_{150}$ & 28,55 & 1,20 & 15,08 & 5,81 & 2,36 & 1,40 \\
\hline $\mathrm{N}_{120} \mathrm{P}_{75} \mathrm{~K}_{150}$ & 25,39 & 1,16 & 16,61 & 6,05 & 2,43 & 1,19 \\
\hline $\mathrm{N}_{120} \mathrm{P}_{300} \mathrm{~K}_{150}$ & 28,36 & 1,45 & 29,48 & 7,53 & 2,78 & 1,32 \\
\hline $\mathrm{N}_{120} \mathrm{P}_{150} \mathrm{~K}_{0}$ & 27,20 & 1,41 & 10,61 & 8,85 & 3,63 & 1,43 \\
\hline $\mathrm{N}_{120} \mathrm{P}_{150} \mathrm{~K}_{75}$ & 25,62 & 1,25 & 12,78 & 7,87 & 2,86 & 1,33 \\
\hline $\mathrm{N}_{120} \mathrm{P}_{150} \mathrm{~K}_{300}$ & 26,16 & 1,15 & 22,96 & 5,26 & 2,14 & 1,05 \\
\hline Média & 27,30 & 1,31 & 18,36 & 7,05 & 2,79 & 1,33 \\
\hline
\end{tabular}


riores aos dos demais (Tabela 4). As variações observadas entre os teores foliares de nutrientes são resultados de diferentes fatores relacionados com o ambiente (solo e clima), com a planta, com a fonte, com a forma de aplicação e com a quantidade de fertilizantes aplicada ao solo. Apesar da necessidade de considerar os efeitos de diluição e de concentração do nutriente na interpretação dos resultados, a padronização da época e do tamanho das folhas coletadas para comporem as amostras, assim como dos demais fatores associados ao controle local do experimento, permitem a assertiva de que as variações observadas são decorrentes de características intrínsecas dos cultivares.

Os teores médios de $\mathrm{N}$ foliar, encontrados para os três cultivares (Tabela 3), são ligeiramente menores, principalmente para os cultivares 'Amanda' e 'Carolina Vitória', do que o valor mínimo da faixa de normalidade, proposta por Lorenzi et al. (1997). Todavia, considerando-se o trata-

Tabela 4. Teores médios de cobre ( $\mathrm{Cu})$, ferro (Fe), manganês $(\mathrm{Mn})$, e zinco $(\mathrm{Zn})$ nas amostras de folhas dos cultivares 'Amanda', 'Carolina Vitória'e 'Duda', coletadas 90 dias após o plantio

\begin{tabular}{|c|c|c|c|c|}
\hline \multirow{3}{*}{ Tratamento } & $\mathrm{Cu}$ & $\mathbf{F e}$ & Mn & $\mathbf{Z n}$ \\
\hline & \multicolumn{4}{|c|}{$\mathrm{mg} \mathrm{kg}^{-1}$} \\
\hline & \multicolumn{4}{|c|}{ 'Amanda' } \\
\hline$\overline{\mathrm{N}_{0} \mathrm{P}_{0} \mathrm{~K}_{0}}$ & 7,16 & 326,66 & 106,99 & 11,48 \\
\hline $\mathrm{N}_{0} \mathrm{P}_{150} \mathrm{~K}_{150}$ & 7,50 & 480,25 & 91,86 & 12,25 \\
\hline $\mathrm{N}_{60} \mathrm{P}_{150} \mathrm{~K}_{150}$ & 8,43 & 163,83 & 133,33 & 11,05 \\
\hline $\mathrm{N}_{120} \mathrm{P}_{150} \mathrm{~K}_{150}$ & 8,49 & 268,09 & 151,00 & 13,14 \\
\hline $\mathrm{N}_{240} \mathrm{P}_{150} \mathrm{~K}_{150}$ & 6,95 & 230,14 & 87,60 & 13,65 \\
\hline $\mathrm{N}_{120} \mathrm{P}_{0} \mathrm{~K}_{150}$ & 9,38 & 373,58 & 101,00 & 14,40 \\
\hline $\mathrm{N}_{120} \mathrm{P}_{75} \mathrm{~K}_{150}$ & 9,24 & 211,66 & 99,66 & 14,08 \\
\hline $\mathrm{N}_{120} \mathrm{P}_{300} \mathrm{~K}_{150}$ & 6,86 & 224,63 & 132,88 & 12,80 \\
\hline $\mathrm{N}_{120} \mathrm{P}_{150} \mathrm{~K}_{0}$ & 7,03 & 407,09 & 109,51 & 11,99 \\
\hline $\mathrm{N}_{120} \mathrm{P}_{150} \mathrm{~K}_{75}$ & 7,58 & 326,56 & 112,04 & 13,76 \\
\hline $\mathrm{N}_{120} \mathrm{P}_{150} \mathrm{~K}_{300}$ & 8,74 & 247,36 & 114,26 & 13,98 \\
\hline \multirow[t]{2}{*}{ Média } & 7,94 & 296,35 & 112,74 & 12,96 \\
\hline & \multicolumn{4}{|c|}{ 'Carolina Vitória' } \\
\hline $\mathrm{N}_{0} \mathrm{P}_{0} \mathrm{~K}_{0}$ & 10,50 & 354,94 & 105,06 & 11,75 \\
\hline $\mathrm{N}_{0} \mathrm{P}_{150} \mathrm{~K}_{150}$ & 9,95 & 290,20 & 108,88 & 11,91 \\
\hline $\mathrm{N}_{60} \mathrm{P}_{150} \mathrm{~K}_{150}$ & 12,49 & 288,83 & 172,10 & 14,38 \\
\hline $\mathrm{N}_{120} \mathrm{P}_{150} \mathrm{~K}_{150}$ & 10,80 & 196,41 & 113,76 & 16,13 \\
\hline $\mathrm{N}_{240} \mathrm{P}_{150} \mathrm{~K}_{150}$ & 11,44 & 140,28 & 123,98 & 14,70 \\
\hline $\mathrm{N}_{120} \mathrm{P}_{0} \mathrm{~K}_{150}$ & 18,08 & 364,09 & 95,54 & 16,03 \\
\hline $\mathrm{N}_{120} \mathrm{P}_{75} \mathrm{~K}_{150}$ & 15,14 & 207,15 & 182,61 & 14,89 \\
\hline $\mathrm{N}_{120} \mathrm{P}_{300} \mathrm{~K}_{150}$ & 10,56 & 171,13 & 115,79 & 14,94 \\
\hline $\mathrm{N}_{120} \mathrm{P}_{150} \mathrm{~K}_{0}$ & 10,85 & 197,90 & 150,69 & 11,81 \\
\hline $\mathrm{N}_{120} \mathrm{P}_{150} \mathrm{~K}_{75}$ & 11,88 & 237,80 & 125,61 & 14,71 \\
\hline $\mathrm{N}_{120} \mathrm{P}_{150} \mathrm{~K}_{300}$ & 11,21 & 151,66 & 97,36 & 14,28 \\
\hline \multirow[t]{2}{*}{ Média } & 12,08 & 236,40 & 126,49 & 14,14 \\
\hline & \multicolumn{4}{|c|}{ 'Duda' } \\
\hline $\mathrm{N}_{0} \mathrm{P}_{0} \mathrm{~K}_{0}$ & 6,61 & 221,83 & 129,53 & 9,45 \\
\hline $\mathrm{N}_{0} \mathrm{P}_{150} \mathrm{~K}_{150}$ & 7,88 & 139,01 & 101,79 & 11,19 \\
\hline $\mathrm{N}_{60} \mathrm{P}_{150} \mathrm{~K}_{150}$ & 8,05 & 124,84 & 77,11 & 12,65 \\
\hline $\mathrm{N}_{120} \mathrm{P}_{150} \mathrm{~K}_{150}$ & 7,45 & 112,61 & 138,44 & 10,34 \\
\hline $\mathrm{N}_{240} \mathrm{P}_{150} \mathrm{~K}_{150}$ & 6,48 & 122,36 & 72,96 & 9,94 \\
\hline $\mathrm{N}_{120} \mathrm{P}_{0} \mathrm{~K}_{150}$ & 10,34 & 315,24 & 100,83 & 12,86 \\
\hline $\mathrm{N}_{120} \mathrm{P}_{75} \mathrm{~K}_{150}$ & 7,56 & 102,15 & 140,86 & 9,73 \\
\hline $\mathrm{N}_{120} \mathrm{P}_{300} \mathrm{~K}_{150}$ & 6,53 & 244,41 & 103,68 & 10,79 \\
\hline $\mathrm{N}_{120} \mathrm{P}_{150} \mathrm{~K}_{0}$ & 7,25 & 185,10 & 100,90 & 10,98 \\
\hline $\mathrm{N}_{120} \mathrm{P}_{150} \mathrm{~K}_{75}$ & 5,98 & 152,71 & 140,75 & 9,79 \\
\hline $\mathrm{N}_{120} \mathrm{P}_{150} \mathrm{~K}_{300}$ & 7,53 & 127,68 & 131,65 & 9,81 \\
\hline Média & 7,42 & 167,99 & 112,59 & 10,68 \\
\hline
\end{tabular}

Rev. Ceres, Viçosa, v. 60, n.6, p. 863-875, nov/dez, 2013 
mento referência $\mathrm{N}_{120} \mathrm{P}_{150} \mathrm{~K}_{150}$, apenas o cultivar 'Duda' apresentou teor inferior ao valor mínimo da faixa de referência. Por outro lado, tanto os teores médios de P e K, quanto os maiores observados, foram significativamente inferiores àqueles considerados.

Por meio da análise de regressão, foi possível observar que, para os três cultivares, os teores foliares de $\mathrm{N}$ e K aumentaram significativamente com as doses de $\mathrm{Ne}_{2} \mathrm{O}$ aplicadas e que, apenas para o cultivar 'Duda', é que os teores de $\mathrm{P}$ aumentaram em função das doses aplicadas de $\mathrm{P}_{2} \mathrm{O}_{5}$ (Figuras 1, 2 e 3). Corroborando as comparações anteriormente feitas, com os resultados apresentados por
Melvin et al. (2002), em lavouras da China, a maior dose de $\mathrm{N}$ utilizada foi insuficiente para atingir a máxima eficiência agronômica, uma vez que, para este nutriente, as equações ajustadas para os três cultivares foram lineares e positivas (Figura 1). Da mesma forma, a maior dose de K utilizada foi insuficiente para o cultivar 'Amanda' (Figura 3 ). Por outro lado, esta resposta pode refletir a menor absorção destes nutrientes, não atingindo um nível de saturação em termos de mecanismos de absorção e, ou, transporte interno da planta. Esta análise é dificultada, uma vez que, foram analisados os teores e não os conteúdos de nutrientes.

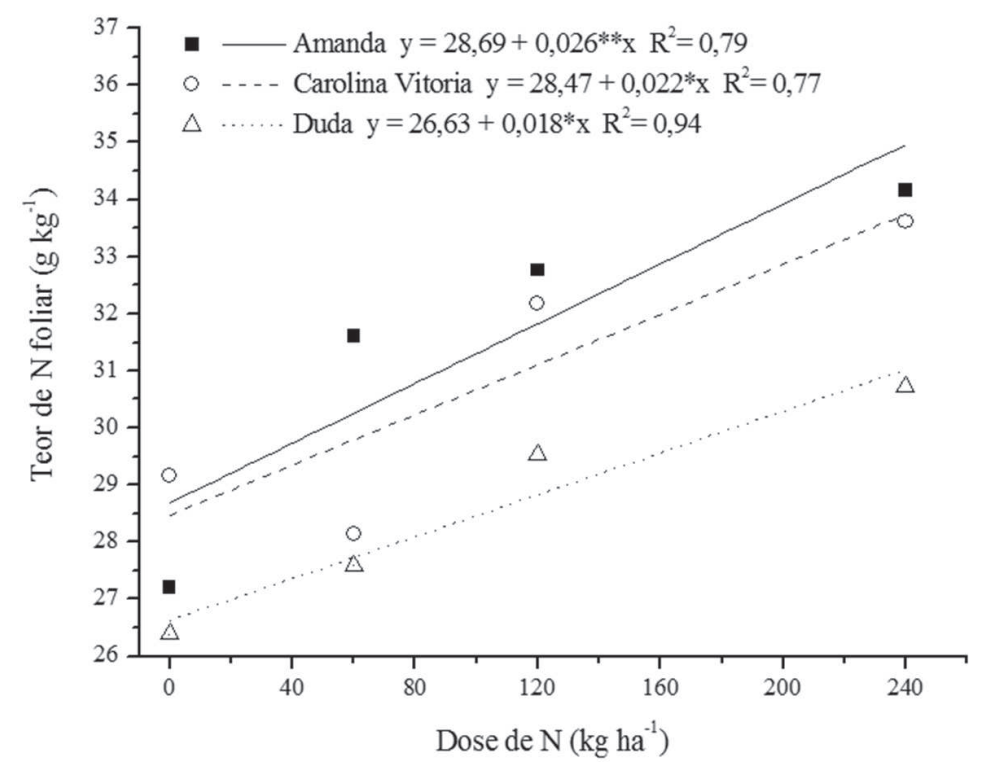

Figura 1. Curvas de resposta para teores foliares de $\mathrm{N}$ de três cultivares de batata-doce, aos 90 dias de cultivo, em função de doses de $\mathrm{N}$ aplicadas ao solo. ${ }^{*}, * *$ Coeficientes significativos a 5 e $1 \%$ de probabilidade pelo teste $\mathrm{F}$, respectivamente.

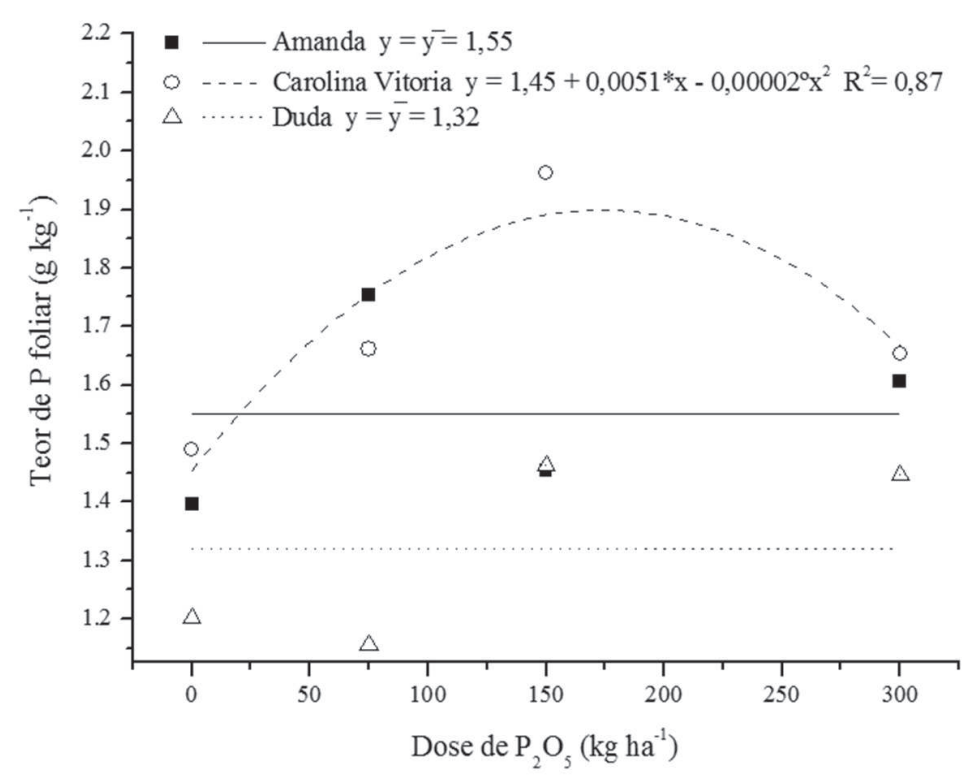

Figura 2. Curvas de resposta para teores foliares de $\mathrm{P}$ de três cultivares de batata-doce, aos 90 dias de cultivo, em função de doses de $\mathrm{P}_{2} \mathrm{O}_{5}$ aplicadas ao solo. ${ }^{\circ} \mathrm{e} *$ Coeficientes significativos a 10 e $5 \%$ de probabilidade pelo teste $\mathrm{F}$, respectivamente. 


\section{Teores de $N, P$ e $K$ nas raizes tuberosas aos 180 dias de cultivo}

Entre os nutrientes analisados nas raízes tuberosas, o $\mathrm{K}$ foi aquele que se apresentou em maiores concentrações, para todos os cultivares (Tabela 5). Considerandose os teores médios entre os tratamentos, os teores de $\mathrm{K}$ foram, em média, $27 \%$ superiores aos de N, o segundo nutriente em maior concentração nas raízes tuberosas, nos cultivares 'Amanda' e 'Carolina Vitória'. Os demais macronutrientes ( $\mathrm{P}, \mathrm{Ca}, \mathrm{Mg}$ e $\mathrm{S}$ ) apresentaram concentrações médias, na mesma ordem de grandeza, e corresponderam, em média, a apenas $6 \%$ da concentração média de K. Como era esperado, os teores de N, P e K nas raízes tuberosas foram significativamente afetados pelas doses de $\mathrm{N}, \mathrm{P}_{2} \mathrm{O}_{5}$ e $\mathrm{K}_{2} \mathrm{O}$ aplicadas, com tendência clara de aumento na concentração com o aumento das doses aplicadas. Essa tendência pode ser mais bem observada por meio dos modelos matemáticos, ajustados para os teores de nutrientes em função das doses aplicadas (Figura 2). Já os teores de $\mathrm{Ca}, \mathrm{Mg}$ e S não variaram estatisticamente, em função dos tratamentos aplicados.

Entre os cultivares, 'Duda' manteve a tendência observada para os teores foliares, apresentando as menores concentrações de macronutrientes em suas raízes tuberosas (Tabela 5). Ao se compararem as concentrações de nutrientes nas raízes tuberosas das plantas cultivadas neste experimento, com as concentrações indicadas por Lorenzi et al. (1979), observa-se que aqueles são significativamente maiores. Esse resultado talvez decorra do fato de as plantas terem sido cultivadas por 180 dias, uma vez que o objetivo foi a maior produção de biomassa para a produção de etanol. Esse longo período, comparado ao tempo de cultivo médio comercial (110-130 dias), pode proporcionar maior translocação interna de nutrientes para as raízes (dreno), o que resultaria em suas maiores concentrações. No entanto, deve-se considerar, da mesma forma, o maior acúmulo de biomassa, o que resultaria em efeito de diluição.

A partir dos teores dos nutrientes nas raízes tuberosas e da produtividade obtida em cada tratamento (Thumé, 2011), foi possível calcular a exportação de nutrientes (Tabela 6). Como o K foi o nutriente com maior concentração nas raízes, suas quantidades exportadas são significativamente superiores às dos demais. Ao se considerarem os valores médios de $\mathrm{K}$ aplicados ao solo, nos diferentes tratamentos (130 $\left.\mathrm{kg} \mathrm{ha}^{-1}\right)$, e as quantidades médias exportadas deste nutriente pelas raízes tuberosas $\left(271 \mathrm{~kg} \mathrm{ha}^{-1}\right)$, verifica-se um balanço negativo de $\mathrm{K}$ no sistema. Esse comportamento evidencia a importância do manejo da adubação potássica, principalmente o parcelamento em solos de textura média e arenosa, para que elevadas produtividades de batata-doce possam ser obtidas. Considerando-se as quantidades médias de nutrientes exportadas pelas raízes tuberosas, em função dos diferentes tratamentos, observa-se que, para os cultivares 'Amanda' e 'Carolina Vitória', a ordem de extração foi $\mathrm{K}>\mathrm{N}>\mathrm{P}>\mathrm{Ca}>$ $\mathrm{Mg}>\mathrm{S}$, enquanto que para o cultivar 'Duda' a ordem foi $\mathrm{K}>\mathrm{N}>\mathrm{Ca}>\mathrm{Mg}>\mathrm{P}>\mathrm{S}$ (Tabela 6).

Contrariamente ao observado neste trabalho, o N (129 kg $\mathrm{ha}^{-1}$ ) foi o nutriente que mais se acumulou nas raízes tuberosas do cultivar Canadense, seguido do $\mathrm{K}\left(81 \mathrm{~kg} \mathrm{ha}^{-1}\right)$ e, este, do Ca (23 kg ha-1) (Echer et al., 2009). Este fato evidencia a necessidade de adequar o manejo da adubação, em relação ao cultivar a ser plantado e ao meio (solo, clima, etc.).

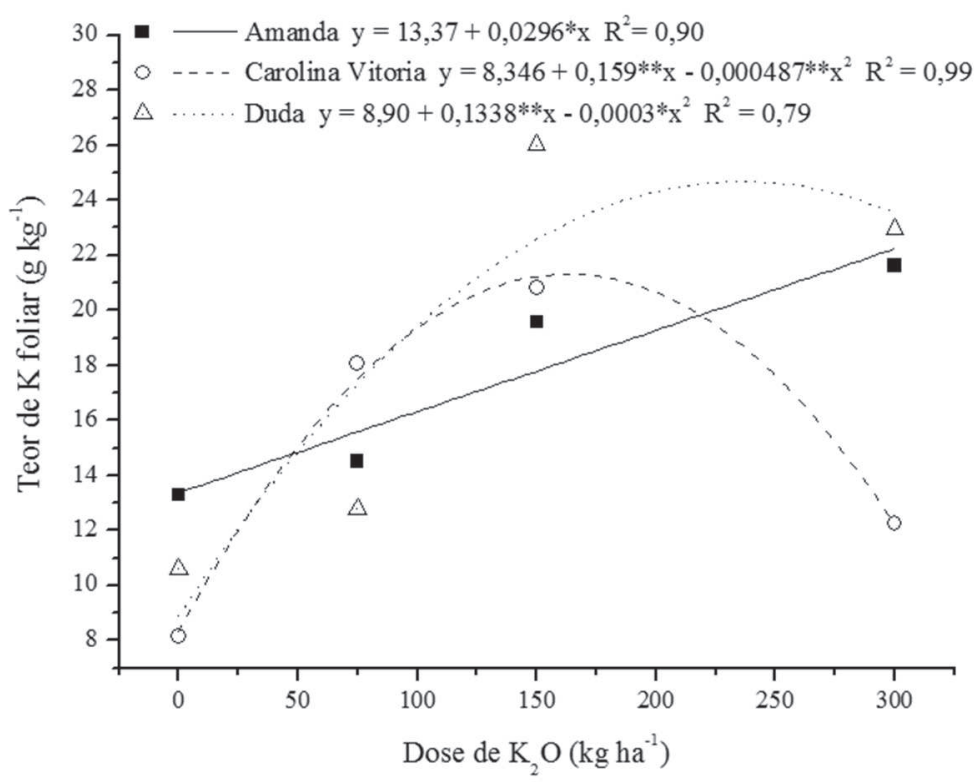

Figura 3. Curvas de resposta para teores foliares de $\mathrm{K}$ de três cultivares de batata-doce, aos 90 dias de cultivo, em função de doses de $\mathrm{N}, \mathrm{P}_{2} \mathrm{O}_{5}$ e $\mathrm{K}_{2} \mathrm{O}$ aplicadas ao solo. *, ** Coeficientes significativos a 5 e $1 \%$ de probabilidade pelo teste $\mathrm{F}$, respectivamente. 
As quantidades potencialmente exportadas de nutrientes pelas raízes tuberosas seguem, obviamente, o mesmo padrão observado para suas concentrações nas raízes. Assim, o $\mathrm{K}$ e o $\mathrm{N}$ foram os nutrientes mais exportados pela cultura da batata-doce, sendo que a magnitude dessa exportação variou fortemente, com as quantidades de fertilizantes aplicadas e, entre os cultivares.
Analisando-se as respostas dos cultivares aos tratamentos aplicados, observa-se que, com exceção do cultivar 'Carolina Vitória', cujo teor médio de $\mathrm{N}$ nas raízes não diferiu significativamente, o cultivar 'Duda' foi aquele que acumulou menos nutrientes nas raízes tuberosas (Figuras 4, 5 e 6); no entanto, apresentou maior produtividade média. Por outro lado, o cultivar 'Amanda' apresentou as maiores exportações de N e K. Provavelmente, os resulta-

Tabela 5. Teores médios de nitrogênio $(\mathrm{N})$, fósforo $(\mathrm{P})$, potássio $(\mathrm{K})$, cálcio $(\mathrm{Ca})$, magnésio $(\mathrm{Mg})$ e enxofre $(\mathrm{S})$ nas amostras de raízes tuberosas dos cultivares 'Amanda', 'Carolina Vitória'e 'Duda', coletadas 180 dias após o plantio, em função de diferentes doses de $\mathrm{N}, \mathrm{P}_{2} \mathrm{O}_{5}$ e $\mathrm{K}_{2} \mathrm{O}$

\begin{tabular}{|c|c|c|c|c|c|c|}
\hline \multirow{3}{*}{ Tratamento } & $\mathbf{N}$ & $\mathbf{P}$ & $\mathbf{K}$ & $\mathrm{Ca}$ & $\mathrm{Mg}$ & $\mathbf{S}$ \\
\hline & \multicolumn{6}{|c|}{$\mathrm{g} \mathrm{kg}^{-1}$} \\
\hline & \multicolumn{6}{|c|}{ 'Amanda' } \\
\hline$\overline{\mathrm{N}_{0} \mathrm{P}_{0} \mathrm{~K}_{0}}$ & 8,48 & 0,74 & 13,73 & 0,66 & 0,69 & 0,56 \\
\hline $\mathrm{N}_{0} \mathrm{P}_{150} \mathrm{~K}_{150}$ & 7,94 & 0,76 & 13,33 & 0,81 & 0,78 & 0,51 \\
\hline $\mathrm{N}_{60} \mathrm{P}_{150} \mathrm{~K}_{150}$ & 7,70 & 0,93 & 11,49 & 0,65 & 0,65 & 0,45 \\
\hline $\mathrm{N}_{120} \mathrm{P}_{150} \mathrm{~K}_{150}$ & 10,71 & 0,97 & 13,13 & 0,90 & 0,74 & 0,60 \\
\hline $\mathrm{N}_{240} \mathrm{P}_{150} \mathrm{~K}_{150}$ & 9,86 & 1,13 & 15,54 & 0,86 & 0,81 & 0,50 \\
\hline $\mathrm{N}_{120} \mathrm{P}_{0} \mathrm{~K}_{150}$ & 8,64 & 0,52 & 12,76 & 0,78 & 0,77 & 0,46 \\
\hline $\mathrm{N}_{120} \mathrm{P}_{75} \mathrm{~K}_{150}$ & 12,48 & 1,28 & 14,35 & 1,08 & 0,91 & 0,67 \\
\hline $\mathrm{N}_{120} \mathrm{P}_{300} \mathrm{~K}_{150}$ & 9,08 & 0,96 & 11,82 & 0,75 & 0,73 & 0,58 \\
\hline $\mathrm{N}_{120} \mathrm{P}_{150} \mathrm{~K}_{0}$ & 9,73 & 0,70 & 10,11 & 0,84 & 0,83 & 0,51 \\
\hline $\mathrm{N}_{120} \mathrm{P}_{150} \mathrm{~K}_{75}$ & 10,56 & 0,85 & 12,91 & 0,79 & 0,80 & 0,51 \\
\hline $\mathrm{N}_{120} \mathrm{P}_{150} \mathrm{~K}_{300}$ & 8,55 & 0,86 & 13,50 & 0,78 & 0,73 & 0,48 \\
\hline \multirow[t]{2}{*}{ Média } & $\mathbf{9 , 4 3}$ & $\mathbf{0 , 8 8}$ & 12,97 & $\mathbf{0 , 8 1}$ & 0,77 & 0,53 \\
\hline & \multicolumn{6}{|c|}{ 'Carolina Vitória' } \\
\hline $\mathrm{N}_{0} \mathrm{P}_{0} \mathrm{~K}_{0}$ & 6,85 & 0,93 & 11,72 & 1,11 & 0,78 & 0,49 \\
\hline $\mathrm{N}_{0} \mathrm{P}_{150} \mathrm{~K}_{150}$ & 7,34 & 0,91 & 12,18 & 0,92 & 0,74 & 0,49 \\
\hline $\mathrm{N}_{60} \mathrm{P}_{150} \mathrm{~K}_{150}$ & 8,05 & 1,10 & 12,85 & 0,92 & 0,79 & 0,49 \\
\hline $\mathrm{N}_{120} \mathrm{P}_{150} \mathrm{~K}_{150}$ & 9,69 & 1,45 & 15,16 & 1,07 & 0,85 & 0,61 \\
\hline $\mathrm{N}_{240} \mathrm{P}_{150} \mathrm{~K}_{150}$ & 8,49 & 1,00 & 12,46 & 0,98 & 0,70 & 0,48 \\
\hline $\mathrm{N}_{120} \mathrm{P}_{0} \mathrm{~K}_{150}$ & 9,04 & 0,92 & 14,00 & 0,95 & 0,79 & 0,49 \\
\hline $\mathrm{N}_{120} \mathrm{P}_{75} \mathrm{~K}_{150}$ & 9,08 & 1,09 & 12,11 & 0,85 & 0,70 & 0,52 \\
\hline $\mathrm{N}_{120} \mathrm{P}_{300} \mathrm{~K}_{150}$ & 8,37 & 0,94 & 10,25 & 0,98 & 0,70 & 0,47 \\
\hline $\mathrm{N}_{120} \mathrm{P}_{150} \mathrm{~K}_{0}$ & 10,90 & 1,28 & 10,23 & 1,27 & 0,95 & 0,63 \\
\hline $\mathrm{N}_{120} \mathrm{P}_{150} \mathrm{~K}_{75}$ & 9,18 & 1,11 & 9,93 & 1,33 & 0,84 & 0,49 \\
\hline $\mathrm{N}_{120} \mathrm{P}_{150} \mathrm{~K}_{300}$ & 9,45 & 1,08 & 13,03 & 1,35 & 1,02 & 0,54 \\
\hline \multirow[t]{2}{*}{ Média } & 8,77 & 1,07 & 12,17 & 1,07 & $\mathbf{0 , 8 1}$ & 0,52 \\
\hline & \multicolumn{6}{|c|}{ 'Duda' } \\
\hline $\mathrm{N}_{0} \mathrm{P}_{0} \mathrm{~K}_{0}$ & 6,70 & 0,45 & 9,26 & 0,59 & 0,56 & 0,30 \\
\hline $\mathrm{N}_{0} \mathrm{P}_{150} \mathrm{~K}_{150}$ & 7,60 & 0,69 & 9,50 & 0,73 & 0,55 & 0,42 \\
\hline $\mathrm{N}_{60} \mathrm{P}_{150} \mathrm{~K}_{150}$ & 8,53 & 0,43 & 7,33 & 0,58 & 0,53 & 0,26 \\
\hline $\mathrm{N}_{120} \mathrm{P}_{150} \mathrm{~K}_{150}$ & 6,72 & 0,52 & 9,32 & 0,70 & 0,59 & 0,27 \\
\hline $\mathrm{N}_{240} \mathrm{P}_{150} \mathrm{~K}_{150}$ & 7,74 & 0,81 & 9,14 & 0,61 & 0,56 & 0,30 \\
\hline $\mathrm{N}_{120} \mathrm{P}_{0} \mathrm{~K}_{150}$ & 8,68 & 0,41 & 10,16 & 0,74 & 0,71 & 0,30 \\
\hline $\mathrm{N}_{120} \mathrm{P}_{75} \mathrm{~K}_{150}$ & 7,60 & 0,51 & 8,97 & 0,87 & 0,65 & 0,36 \\
\hline $\mathrm{N}_{120} \mathrm{P}_{300} \mathrm{~K}_{150}$ & 6,04 & 0,61 & 7,35 & 0,55 & 0,47 & 0,29 \\
\hline $\mathrm{N}_{120} \mathrm{P}_{150} \mathrm{~K}_{0}$ & 8,31 & 0,49 & 5,44 & 0,74 & 0,67 & 0,26 \\
\hline $\mathrm{N}_{120} \mathrm{P}_{150} \mathrm{~K}_{75}$ & 6,80 & 0,57 & 8,95 & 0,62 & 0,49 & 0,35 \\
\hline $\mathrm{N}_{120} \mathrm{P}_{150} \mathrm{~K}_{300}$ & 7,68 & 0,42 & 8,29 & 0,93 & 0,75 & 0,34 \\
\hline Média & 7,49 & 0,54 & 8,52 & $\mathbf{0 , 7 0}$ & 0,59 & 0,31 \\
\hline
\end{tabular}


dos encontrados refletem características intrínsecas de cada cultivar, indicando, desta maneira, que as adubações com esses nutrientes devem ser individualizadas, em função do cultivar, para obter-se maior eficiência econômica e ambiental.

\section{Teores críticos foliares de $\mathrm{N}, \mathrm{P}$ e $\mathrm{K}$}

A partir da obtenção das doses recomendadas, de cada nutriente, por meio das equações de regressão, ajustadas para produtividade de raízes tuberosas, em função das doses de $\mathrm{N}, \mathrm{P}_{2} \mathrm{O}_{5}$ e $\mathrm{K}_{2} \mathrm{O}$ (Thumé, 2011), foram obtidos os níveis críticos foliares de N, P e K, para cada cultivar (Tabela 7). O nível crítico pode ser definido como um valor que separa plantas deficientes daquelas não deficientes, ou ainda, o teor mínimo na planta que proporciona produtividade equivalente a $90 \%$ da produtividade máxima (Wadt \& Novais, 1999). Também pode ser entendido como aquela concentração foliar do nutriente, em relação à qual

Tabela 6. Quantidades médias de macronutrientes exportadas pelas raízes tuberosas dos cultivares 'Amanda', 'Carolina Vitória' e 'Duda', aos 180 dias após o plantio, em função dos diferentes tratamentos

\begin{tabular}{|c|c|c|c|c|c|c|}
\hline \multirow{3}{*}{ Tratamento } & $\mathbf{N}$ & $\mathbf{P}$ & $\mathbf{K}$ & $\mathbf{C a}$ & Mg & $\mathbf{S}$ \\
\hline & \multicolumn{6}{|c|}{$\mathrm{kg} \mathrm{ha}^{-1}$} \\
\hline & \multicolumn{6}{|c|}{ 'Amanda' } \\
\hline$\overline{\mathrm{N}_{0} \mathrm{P}_{0} \mathrm{~K}_{0}}$ & 115,16 & 10,05 & 186,45 & 8,96 & 9,37 & 7,60 \\
\hline $\mathrm{N}_{0} \mathrm{P}_{150} \mathrm{~K}_{150}$ & 109,02 & 10,43 & 183,02 & 11,12 & 10,71 & 7,00 \\
\hline $\mathrm{N}_{60} \mathrm{P}_{150} \mathrm{~K}_{150}$ & 149,53 & 18,06 & 223,14 & 12,62 & 12,62 & 8,74 \\
\hline $\mathrm{N}_{120} \mathrm{P}_{150} \mathrm{~K}_{150}$ & 251,15 & 22,75 & 307,90 & 21,11 & 17,35 & 14,07 \\
\hline $\mathrm{N}_{240} \mathrm{P}_{150} \mathrm{~K}_{150}$ & 280,81 & 32,18 & 442,58 & 24,49 & 23,07 & 14,24 \\
\hline $\mathrm{N}_{120} \mathrm{P}_{0} \mathrm{~K}_{150}$ & 163,56 & 9,84 & 241,55 & 14,77 & 14,58 & 8,71 \\
\hline $\mathrm{N}_{120} \mathrm{P}_{75} \mathrm{~K}_{150}$ & 239,37 & 24,55 & 275,23 & 20,71 & 17,45 & 12,85 \\
\hline $\mathrm{N}_{120} \mathrm{P}_{300} \mathrm{~K}_{150}$ & 221,37 & 23,40 & 288,17 & 18,29 & 17,80 & 14,14 \\
\hline $\mathrm{N}_{120} \mathrm{P}_{150} \mathrm{~K}_{0}$ & 156,17 & 11,24 & 162,27 & 13,48 & 13,32 & 8,19 \\
\hline $\mathrm{N}_{120} \mathrm{P}_{150} \mathrm{~K}_{75}$ & 246,36 & 19,83 & 301,19 & 18,43 & 18,66 & 11,90 \\
\hline $\mathrm{N}_{120} \mathrm{P}_{150} \mathrm{~K}_{300}$ & 185,62 & 18,67 & 293,09 & 16,93 & 15,85 & 10,42 \\
\hline \multirow[t]{2}{*}{ Média } & 196,80 & 18,40 & 270,68 & 16,89 & 16,01 & 11,06 \\
\hline & \multicolumn{6}{|c|}{ 'Carolina Vitória' } \\
\hline $\mathrm{N}_{0} \mathrm{P}_{0} \mathrm{~K}_{0}$ & 101,52 & 13,78 & 173,69 & 16,45 & 11,56 & 7,26 \\
\hline $\mathrm{N}_{0} \mathrm{P}_{150} \mathrm{~K}_{150}$ & 127,42 & 15,80 & 211,44 & 15,97 & 12,85 & 8,51 \\
\hline $\mathrm{N}_{60} \mathrm{P}_{150} \mathrm{~K}_{150}$ & 156,73 & 21,42 & 250,19 & 17,91 & 15,38 & 9,54 \\
\hline $\mathrm{N}_{120} \mathrm{P}_{150} \mathrm{~K}_{150}$ & 196,61 & 29,42 & 307,60 & 21,71 & 17,25 & 12,38 \\
\hline $\mathrm{N}_{240} \mathrm{P}_{150} \mathrm{~K}_{150}$ & 195,61 & 23,04 & 287,08 & 22,58 & 16,13 & 11,06 \\
\hline $\mathrm{N}_{120} \mathrm{P}_{0} \mathrm{~K}_{150}$ & 138,76 & 14,12 & 214,90 & 14,58 & 12,13 & 7,52 \\
\hline $\mathrm{N}_{120} \mathrm{P}_{75} \mathrm{~K}_{150}$ & 174,15 & 20,91 & 232,27 & 16,30 & 13,43 & 9,97 \\
\hline $\mathrm{N}_{120} \mathrm{P}_{300} \mathrm{~K}_{150}$ & 193,26 & 21,70 & 236,67 & 22,63 & 16,16 & 10,85 \\
\hline $\mathrm{N}_{120} \mathrm{P}_{150} \mathrm{~K}_{0}$ & 147,15 & 17,28 & 138,11 & 17,15 & 12,83 & 8,51 \\
\hline $\mathrm{N}_{120} \mathrm{P}_{150} \mathrm{~K}_{75}$ & 163,22 & 19,74 & 176,56 & 23,65 & 14,94 & 8,71 \\
\hline $\mathrm{N}_{120} \mathrm{P}_{150} \mathrm{~K}_{300}$ & 161,03 & 18,40 & 222,03 & 23,00 & 17,38 & 9,20 \\
\hline \multirow[t]{2}{*}{ Média } & 163,21 & 19,98 & 226,57 & 19,85 & 14,99 & 9,64 \\
\hline & \multicolumn{6}{|c|}{ 'Duda' } \\
\hline $\mathrm{N}_{0} \mathrm{P}_{0} \mathrm{~K}_{0}$ & 82,68 & 5,55 & 114,27 & 7,28 & 6,91 & 3,70 \\
\hline $\mathrm{N}_{0} \mathrm{P}_{150} \mathrm{~K}_{150}$ & 186,73 & 16,95 & 233,42 & 17,94 & 13,51 & 10,32 \\
\hline $\mathrm{N}_{60} \mathrm{P}_{150} \mathrm{~K}_{150}$ & 220,93 & 11,14 & 189,85 & 15,02 & 13,73 & 6,73 \\
\hline $\mathrm{N}_{120} \mathrm{P}_{150} \mathrm{~K}_{150}$ & 185,81 & 14,38 & 257,70 & 19,36 & 16,31 & 7,47 \\
\hline $\mathrm{N}_{240} \mathrm{P}_{150} \mathrm{~K}_{150}$ & 216,57 & 22,66 & 255,74 & 17,07 & 15,67 & 8,39 \\
\hline $\mathrm{N}_{120} \mathrm{P}_{0} \mathrm{~K}_{150}$ & 111,45 & 5,26 & 130,45 & 9,50 & 9,12 & 3,85 \\
\hline $\mathrm{N}_{120} \mathrm{P}_{75} \mathrm{~K}_{150}$ & 146,98 & 9,86 & 173,48 & 16,83 & 12,57 & 6,96 \\
\hline $\mathrm{N}_{120} \mathrm{P}_{300} \mathrm{~K}_{150}$ & 164,53 & 16,62 & 200,21 & 14,98 & 12,80 & 7,90 \\
\hline $\mathrm{N}_{120} \mathrm{P}_{150} \mathrm{~K}_{0}$ & 96,40 & 5,68 & 63,10 & 8,58 & 7,77 & 3,02 \\
\hline $\mathrm{N}_{120} \mathrm{P}_{150} \mathrm{~K}_{75}$ & 842,86 & 13,65 & 214,35 & 14,85 & 11,74 & 8,38 \\
\hline $\mathrm{N}_{120} \mathrm{P}_{150} \mathrm{~K}_{300}$ & 195,61 & 10,70 & 211,15 & 23,69 & 19,10 & 8,66 \\
\hline Média & 169,65 & 12,17 & 192,96 & 15,77 & 13,45 & 7,10 \\
\hline
\end{tabular}




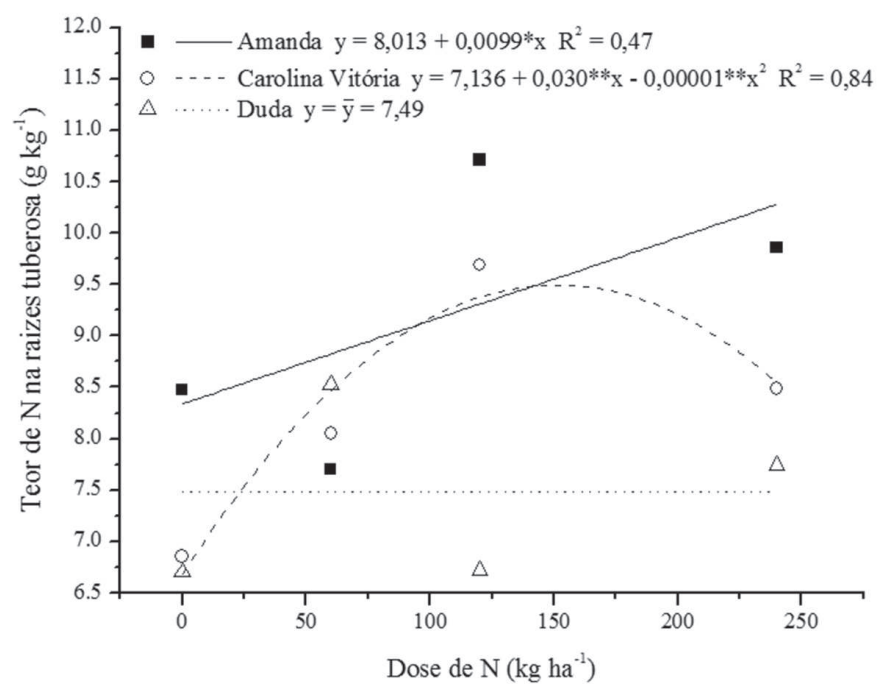

Figura 4. Curvas de resposta para teores de $\mathrm{N}$ nas raízes tuberosas de três cultivares de batata-doce, aos 180 dias de cultivo, em função de diferentes doses de N. *, ** Coeficientes significativos a 5 e $1 \%$ de probabilidade pelo teste F, respectivamente.

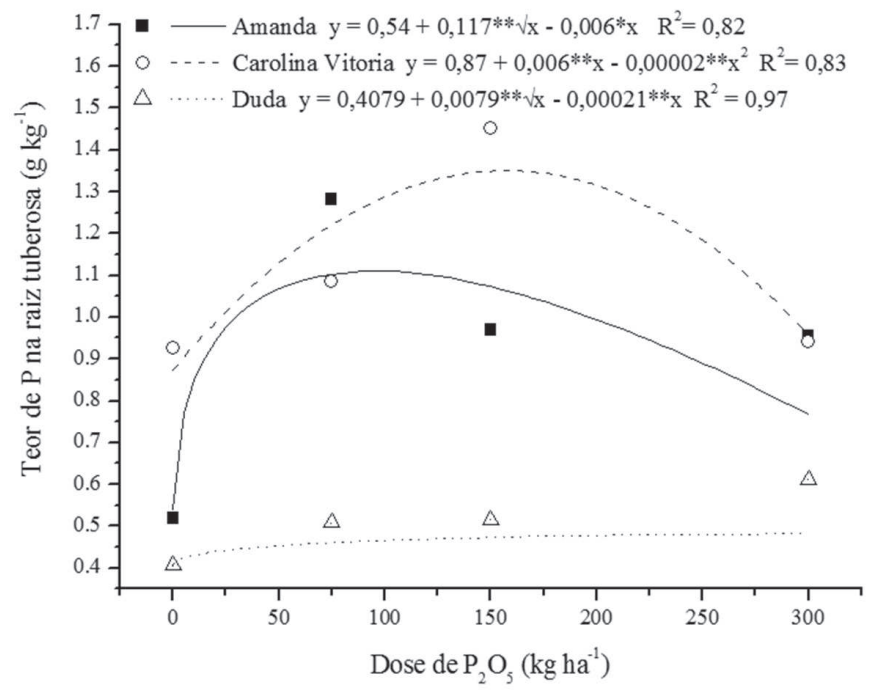

Figura 5. Curvas de resposta para teores de $\mathrm{P}$ nas raízes tuberosas de três cultivares de batata-doce, aos 180 dias de cultivo, em função de diferentes doses de $\mathrm{P}_{2} \mathrm{O}_{5}$. * ** Coeficientes significativos a 5 e $1 \%$ de probabilidade pelo teste $\mathrm{F}$, respectivamente.

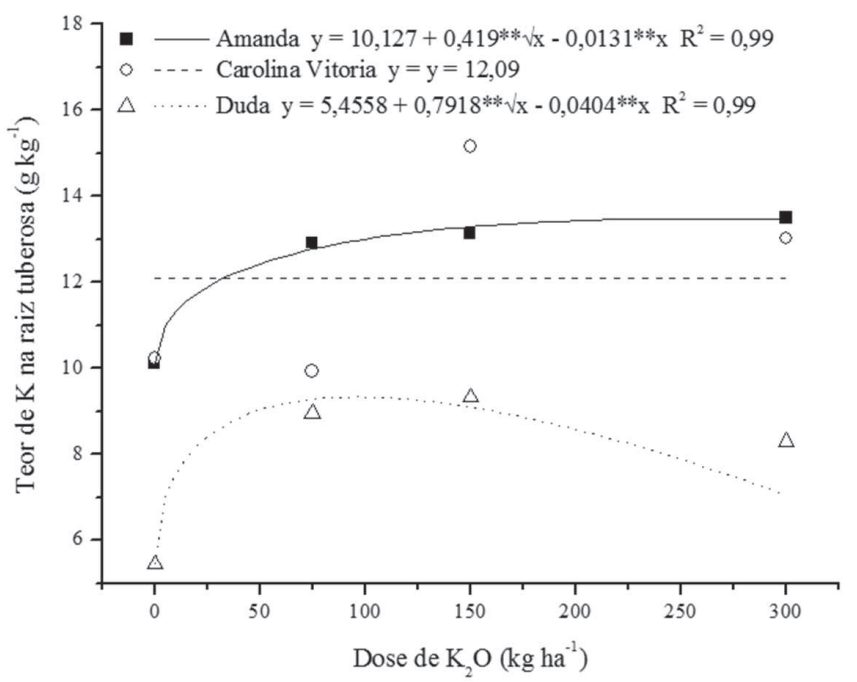

Figura 6. Curvas de resposta para teores de K nas raízes tuberosas de três cultivares de batata-doce, aos 180 dias de cultivo, em função de diferentes doses de $\mathrm{K}_{2} \mathrm{O}$. ** Coeficientes significativos a $1 \%$ de probabilidade pelo teste $\mathrm{F}$, respectivamente. 
Tabela 7. Níveis críticos de N, Pe K nas folhas de três cultivares de batata-doce aos 90 dias após o plantio

\begin{tabular}{lccc}
\hline & \multicolumn{3}{c}{ Nível crítico foliar } \\
\cline { 2 - 4 } Cultivar & $\mathbf{N}$ & $\mathbf{P}$ & $\mathbf{K}$ \\
\cline { 2 - 4 } & \multicolumn{3}{c}{$\mathrm{g} \mathrm{kg}^{-1}$} \\
\hline 'Amanda' & 33,1 & $*$ & 14,9 \\
'Carolina Vitória' & 31,5 & 1,8 & 18,2 \\
'Duda' & 27,5 & $*$ & 20,5 \\
\hline
\end{tabular}

* Valor não obtido em função dos dados não permitirem o ajuste de uma equação (entre os modelos testados) entre os teores foliares e as doses de $\mathrm{P}_{2} \mathrm{O}_{5}$ aplicadas ao solo.

concentrações inferiores significam alta probabilidade de resposta positiva à adição do nutriente, ou que concentrações acima significam baixa probabilidade de resposta positiva à adição do nutriente.

O nível crítico foliar de $\mathrm{N}$, observado para o cultivar 'Duda', foi inferior aos observados para os demais cultivares. Por outro lado, esse mesmo cultivar apresentou o nível crítico foliar de $\mathrm{K}$ superior ao dos demais cultivares (Tabela 7). Em função da impossibilidade de se ajustar um modelo matemático (entre os testados) para os teores foliares de P, para os cultivares "Amanda' e "Duda", foi possível obter o nível crítico foliar de $\mathrm{P}$ apenas para o cultivar 'Carolina Vitória' $\left(1,8 \mathrm{~g} \mathrm{~kg}^{-1}\right)$.

A ausência de referências, na literatura, sobre níveis críticos para a batata-doce, impossibilita a comparação dos resultados obtidos neste trabalho. No entanto, em relação à faixa de normalidade proposta por Lorenzi et al. (1997), os níveis críticos de N, observados neste trabalho, situam-se ligeiramente abaixo do limite inferior da fai$\mathrm{xa}$, enquanto os de $\mathrm{K}$ foram muito menores que o limite inferior proposto.

Considerando-se os níveis críticos no solo (Thumé, 2011) e foliares, obtidos neste trabalho, as exigências nutricionais dos cultivares obedeceria a seguinte sequência: N: 'Amanda' > 'Carolina Vitória'> 'Duda'; P: 'Amanda' > 'Duda'> 'Carolina Vitória', e K: 'Duda'> ‘Carolina Vitória'> 'Amanda'. Desta maneira, sugere-se que as adubações com esses nutrientes devem ser individualizadas, em função do cultivar, para que maior eficiência econômica e ambiental possa ser atingida.

\section{CONCLUSÕES}

Os teores foliares de N, S, Cu e Zn variaram apenas em função dos cultivares, enquanto os teores foliares de $\mathrm{P}$ e $\mathrm{K}$ variaram em função das doses de $\mathrm{P}_{2} \mathrm{O}_{5}$ e $\mathrm{K}_{2} \mathrm{O}$, respectivamente, aplicadas ao solo.

Os teores de $\mathrm{N}$, P e $\mathrm{K}$ nas raízes tuberosas variaram significativamente em função das doses desses nutrientes, sendo que o $\mathrm{K}$ foi o nutriente que se acumulou em maior quantidade nesse compartimento das plantas, seguido do $\mathrm{N}$ e, este, de $\mathrm{Ca}, \mathrm{P}, \mathrm{Mg}$ e $\mathrm{S}$.
De acordo com os níveis críticos foliares obtidos, o cultivar 'Amanda' mostrou-se mais exigente em $\mathrm{N}$ e $\mathrm{P}$ e, 'Duda', em K.

\section{AGRADECIMENTOS}

Os autores agradecem a revisão realizada pela Dra. Ecila Mercês de Albuquerque Villani, bolsista de Pósdoutoramento do Departamento de Solos da UFV (Bolsa FAPEMIG) e à CAPES, pela bolsa de mestrado para o primeiro autor.

\section{REFERÊNCIAS}

Alves AU, Oliveira AP, Alves EU, Oliveira ANP, Cardoso EA \& Matos BF (2009) Manejo da adubação nitrogenada para a batata-doce: fontes e parcelamento da aplicação. Ciência e Agrotecnologia, 33:1554-1559.

Bailey JS, Ramakrishna A \& Kirchhof G (2009) An evaluation of nutritional constraints on sweet potato (Ipomea batatas) production in the central highlands of Papua New Guinea. Plant and Soil, 316:97-105.

Beaufils ER (1973) Diagnosis and recommendation integrated system (DRIS). A general scheme of experimentation based on principles developed from research in plant nutrition. Pietermaritzburg, University of Natal. 132p. (Soil Science Bulletin, 1).

Bremner JM \& Mulvaney CS (1982) Nitrogen total. In: Page AL (Ed.) Methods of soil analysis. Madison, American Society of Agronomy. p.595-624.

Echer FR, Dominato JC \& Creste JE (2009) Absorção de nutrientes e distribuição da massa fresca e seca entre órgãos de batatadoce. Horticultura Brasileira, 27:176-182.

Embrapa - Empresa Brasileira de Pesquisa Agropecuária (1997) Cultivo da batata-doce (Ipomoea batatas (L.) Lam.). $3^{\text {a }}$ ed. Brasília, Centro Nacional de Pesquisas de Hortaliças. 18p. (Instruções Técnicas, 7).

Ferreira ME, Castellane PD \& Cruz MCP (1990) Nutrição e adubação de hortaliças. Simpósio sobre nutrição e adubação de hortaliças. Jaboticabal, Potafós. 480p.

Foloni JSS, Corte AJ, Corte JRN, Echer FR \& Tiritan CS (2013) Adubação de cobertura na batata-doce com doses combinadas de nitrogênio e potássio. Semina Ciências Agrárias, 34:117-126.

Jones JBJr, Wolf B \& Mills HA (1991) Plant Analysis handbook. A practical samplying, preparation, analysis, and interpretation guide. Georgia, Micro-Macro Publishing. 213p.

Lorenzi JO, Monteiro DA \& Miranda Filho HS (1997) Raízes e tubérculos. In: Raij B Van, Cantarella H, Quaggio JA \& Furlani AMC (Eds.) Recomendação de adubação e calagem para o estado de São Paulo. 2a ed. Campinas, IAC. p.221-230. (Boletim Técnico, 100).

Melvin SG, Guoquan L \& Weijun Z (2002) Genotypic variation for potassium uptake and utilization efficiency in sweet potato (Ipomea batatas L.). Fields Crop Research, 77:07-15.

Oliveira AP, Oliveira MRT, Barbosa JA, Silva GG, Nogueira DH, Moura MF \& Braz MS (2005) Rendimento e qualidade de raízes de batata-doce adubada com níveis de ureia. Horticultura Brasileira, 23:925-928.

Oliveira AP, Moura MF, Nogueira DH, Chagas NG, Braz MSS, Oliveira MRT \& Barbosa JA (2006) Produção de raízes de batata-doce em função do uso de doses de $\mathrm{N}$ aplicadas no solo e via foliar. Horticultura Brasileira, 24:279-282. 
Oliveira AP, Barbosa AHD, Cavalcante LF, Pereira WE \& Oliveira ANP (2007) Produção de batata-doce adubada com esterco bovino e biofertilizante. Ciência e Agrotecnologia, 31:17221728 .

Oliveira AP, Santos JF, Cavalcante LF, Pereira WE, Santos MCCA Oliveira ANP \& Silva NV (2010) Yield of sweet potato fertilized with cattle manure and biofertilizer. Horticultura Brasileira, 28:277-281.

Ramakrishna A, Bailey JS \& Kirchhof G (2009) A preliminary diagnosis and recommendation integratedsystem (DRIS) model for diagnosing the nutrient status of sweet potato (Ipomoea batatas). Plant Soil, 316:107-116.

Santos JF, Brito CH \& Santos MCCA (2010) Avaliação da produção de batata-doce em função de níveis de adubação orgânica. Acta Scientiarum Agronomy, 32:663-666.

Silva JBC, Lopes CA \& Magalhães JS (2002) Cultura da batatadoce. In: Cereda MP (Ed.) Agricultura: tuberosas amiláceas latino americanas. São Paulo, Cargil. p.449-503.

Silveira MA, André CMG, Alvim TC, Dias LE, Tavares IB, Santana WR \& Souza FR (2007) A cultura da batata-doce como fonte de matéria-prima para a produção de etanol. Palmas, Universidade Federal do Tocantins. 45p. (Boletim Técnico).
Sumner ME (1977) Effect of corn leaf sampled on N, P, K, Ca and $\mathrm{Mg}$ content and calculated DRIS indices. Communication in Soil Science and Plant Analysis, 8:269-280.

Thumé MA (2011) Produtividade e absorção de nutrientes de três cultivares de batata-doce [Ipomoea batatas (L.) Lam] selecionadas para a produção de etanol, em função de diferentes doses de N, P e K aplicados no solo. Dissertação de mestrado. Universidade Federal do Tocantins, Palmas. 58p.

Turrent FA (1979) Uso de una matriz mixta para la optimizacion de cinco a ocho factores controlables de la produccion. Chapingo, Colegio de postgraduados. $65 \mathrm{p}$.

Wadt PGS \& Novais RF (1999) O monitoramento nutricional frente aos métodos diagnósticos no planejamento das adubações. In: Simpósio sobre Monitoramento Nutricional para a Recomendação de Adubação de Culturas, Piracicaba. Anais, Potafos. p.01-18. 